Pacific Journal of Mathematic 


\title{
COMPLETENESS OF SETS OF TRANSLATED COSINES
}

\author{
R. P. BOAs, JR.
}

1. Introduction. Conditions for the completeness on $(0, \pi)$ of sets $\left\{\cos \lambda_{n} x\right\}$ are well known. Ilere we shall consider sets $\left\{\cos \left(\lambda_{n} x+q_{n}\right)\right\}$. Such sets seem first to have been considered by Ditkin [3], who proved that $\left\{\cos \left(n x+q_{n}\right)\right\}_{0}^{\infty}$ is $L$-complete in $(0, \pi)$ if $0 \leq q_{n}<\pi / 2$.

Ditkin's very simple proof uses Fourier series and does not seem capable of extension to the more general sets considered here. Our principal object is to show how the problem may be attacked by complex-variable methods; we shall not attempt an exhaustive discussion.

As a specimen we quote the following case. If $\lambda_{n} \geq 0$ and $\left|\lambda_{n}-n\right| \leq$ $\delta<1 / 2$, then the sets $\left\{\cos \left(\lambda_{n} x+q_{n}\right)\right\}_{0}^{\infty}$ and $\left\{\sin \left(\lambda_{n} x+q_{n}\right)\right\}_{1}^{\infty}$ are $L-$ complete in $(0, \pi)$ if $\pi \delta / 2 \leq q_{n}<\pi(1-\delta) / 2$. (The statement " $\left\{f_{n}(x)\right\}$ is $L^{p}$-complete" means that the only functions of $L^{p}$ which are orthogonal to all $f_{n}(x)$ are almost everywhere zero.) A further result, not covered by the present paper, has been given by Bitsadze [1], who showed that every function satisfying a hölder condition admits a uniformly convergent expansion in terms of the set $\{\cos (n x+\pi / 4)\}$; he indicates an application of this result to the Tricomi partial differential equation.

We remark that although Ditkin's set $\left\{\cos \left(n x+q_{n}\right)\right\}_{0}^{\infty}$ remains complete when all $q_{n}=\pi / 2$, it may fail to be complete if some but not all $q_{n}=\pi / 2$. In fact, the set $\{l, \sin x, \cos 2 x, \cos 3 x, \cdots\}$ is orthogonal to $\cos x$. However, we shall show that not only is the set $\left\{\sin \left(n x+q_{n}\right)\right\}_{0}^{\infty}$ complete if $0 \leq q_{n}<\pi / 2$, but even the set $\left\{\sin \left(n x+q_{n}\right)\right\}_{1}^{\infty}$ is complete.

By applying the completeness theorem of Paley and Wiener $[5, \mathrm{p} .100]$ to the equivalent set $\left\{\cos n x+a_{n} \sin n x\right\}, 0 \leq\left|a_{n}\right|<1$, we can show at once that $\left\{\cos \left(n x+q_{n}\right)\right\}_{0}^{\infty}$ is $L^{2}$-complete if either $0 \leq\left|q_{n}\right| \leq \delta<\pi / 4$ for all $n$ or else $\pi / 4<\delta \leq\left|q_{n}\right| \leq \pi / 2$ for all $n$. The problem of necessary and sufficient conditions for the completeness of $\left\{\cos \left(n x+q_{n}\right)\right\}$ remains open.

2. A general theorem. We shall obtain our results on $\left\{\cos \left(\lambda_{n} x+q_{n}\right)\right\}$ as

Received December 4, 1950.

Pacific J. Math. 1 (1951), 321-328. 
corollaries of a theorem on a related set of more artificial appearance.

THEOREM. Let $\left\{\lambda_{n}\right\}_{0}^{\infty}$ be an increasing unbounded sequence of nonnegative numbers; let $N_{1}(r)$ and $N_{2}(r)$ denote respectively the number of $\lambda_{2 n}$ and of $\lambda_{2 n+1}$ not exceeding $r$. If both

$$
\int_{1}^{r} t^{-1} N_{1}(t) d t>\frac{1}{2} r-\gamma \log r-\text { constant, }
$$

and

$$
\int_{1}^{r} t^{-1} N_{2}(t) d t>\frac{1}{2} r-\left(\gamma+\frac{1}{2}\right) \log r-\text { constant }
$$

where $\gamma=1 /\left(2 p^{\prime}\right)$ if $1 \leq p<\infty, p^{\prime}=p /(p-1)$, and $\gamma<1 / 2$ if $p=\infty$, then the set

$$
\left\{\begin{array}{l}
\cos \lambda_{2 n} t+a_{2 n} \sin \lambda_{2 n} t, \\
-a_{2 n+1} \cos \lambda_{2 n+1} t+\sin \lambda_{2 n+1} t
\end{array}\right.
$$

is $L^{p}$-complete on $(-\pi / 2, \pi / 2)$ if the $a_{n}$ are real numbers all of the same sign.

COROLLARY 1 . The set (2.3), with the $a_{n}$ all of the same sign, is $L^{p}$-complete on $(-\pi / 2, \pi / 2)$ if $0 \leq \lambda_{n} \leq n+1+1 / p^{\prime}, 1 \leq p<\infty$; it is $L^{\infty}$-complete if $0 \leq \lambda_{n} \leq n+\delta, \delta<2$.

Corollary 2. If $\lambda_{n} \geq 0$ and

$$
\left|\lambda_{n}-{ }_{n}\right| \leq \delta<\frac{1}{2}, \frac{\pi \delta}{2} \leq q_{n}<\frac{\pi(1-\delta)}{2},
$$

then the set $\left\{\cos \left(\lambda_{n} x+q_{n}\right)\right\}_{0}^{\infty}$ is L-complete on $(0, \pi)$.

For $\delta=0$, Corollary 2 reduces to Ditkin's theorem; for $\delta \neq 0$, the range of $q_{n}$ is more restricted. If the $\lambda_{n}$ are confined to one side of $n$, a sharper result is true.

CoROLLARY 3. If $n \leq \lambda_{n} \leq n+\delta, 0 \leq \delta<1$, and $0 \leq q_{n}<\pi(1-\delta) / 2$, $n \geq 0$; or if $n-\delta \leq \lambda_{n} \leq n$ for $n>0,0 \leq \delta<1$, and $\pi(1-\delta) / 2<q_{n} \leq 0$, then $\left\{\cos \left(\lambda_{n} x+q_{n}\right)\right\}_{0}^{\infty}$ is L-complete in $(0, \pi)$.

The following result on sets of sines includes the fact that $\left\{\sin \left(n x+q_{n}\right)\right\}_{1}^{\infty}$ 
is $L$-complete on $(0, \pi)$ if $0 \leq q_{n}<\pi / 2$.

Corollary 4. If $\left|n+1-\lambda_{n}\right| \leq \delta<1 / 2$ and $\pi \delta / 2 \leq q_{n}<\pi(1-\delta) / 2$, then the set $\left\{\sin \left(\lambda_{n} x+q_{n}\right)\right\}_{0}^{\infty}$ is L-complete on $(0, \pi)$.

By demanding only $L^{p}$-completeness instead of $L$-completeness, we can allow the $\lambda_{n}$ to be larger than in Corollary 2 .

CorollaRy 5. If $1<p<\infty$ and $n+2-\delta<\lambda_{n}<n+2-1 / p, 1 / p<$ $\delta<1$, then the set $\left\{\cos \left(\lambda_{n} x+q_{n}\right)\right\}_{0}^{\infty}$ is $L^{p}$-complete on $(0, \pi)$ if $\pi \delta / 2 \leq$ $q_{n}<\pi / 2$.

3. Proof of the general theorem. We now prove the theorem stated above. We must show that if $f(x) \in L^{\mathrm{p}}$ and if

$$
\begin{aligned}
& \int_{-\pi / 2}^{\pi / 2}\left(\cos \lambda_{2 n} t+a_{2 n} \sin \lambda_{2 n} t\right) f(t) d t \\
& =\int_{-\pi / 2}^{\pi / 2}\left(-a_{2 n+1} \cos \lambda_{2 n+1} t+\sin \lambda_{2 n+1} t\right) f(t) d t \\
& =0 \quad(n=0,1,2, \cdots),
\end{aligned}
$$

where all $a_{n}$ satisfy $a_{n} \geq 0$ or else all $a_{n}$ satisfy $a_{n} \leq 0$, then $f(x)=0$ almost everywhere.

Write

$$
F(z)=\int_{-\pi / 2}^{\pi / 2} f(t) \cos z t d t, \quad G(z)=\int_{-\pi / 2}^{\pi / 2} f(t) \sin z t d t
$$

then $(3.1)$ is

$$
\begin{gathered}
F\left(\lambda_{2 n}\right)+a_{2 n} G\left(\lambda_{2 n}\right)=0, \\
-a_{2 n+1} F\left(\lambda_{2 n+1}\right)+G\left(\lambda_{2 n+1}\right)=0 .
\end{gathered}
$$

Let $H(z)=F(z) G(z)$; then $H(0)=0$; if $\lambda_{0}=0$, then $H^{\prime}(0)=H^{\prime \prime}(0)=0$; and $H\left(\lambda_{2 n}\right) H\left(\lambda_{2 n+1}\right) \leq 0$. Note that $H(z)$ is an odd function. Let $N(t)=N_{1}(t)+$ $N_{2}(t)$, and let $\Lambda(t)$ denote the number of zeros of $H(z)$ in $0 \leq|z| \leq t$.

We prove first that

$$
\Lambda(r) \geq 2 N(r)+1
$$


To begin with, if $\lambda_{0}=0$, we have, for $0 \leq r<\lambda_{1}$, the relations $N(t)=1$, $\Lambda(r) \geq 3$; if $\lambda_{0}>0$, we have $N(t)=0$ for $0 \leq r<\lambda_{0}, \Lambda(r)=1$. We proceed by induction. Suppose that (3.4) is true for $r \leq \lambda_{k}$. Then it remains true for $r<\lambda_{k+1}$, since $N(r)$ does not change in $\lambda_{k} \leq r<\lambda_{k+1}$. If $H\left(\lambda_{k}\right) H\left(\lambda_{k+1}\right) \neq 0$, then $H\left(\lambda_{k}\right)$ and $H\left(\lambda_{k+1}\right)$ have opposite signs and so $\Lambda\left(\lambda_{k+1}\right) \geq \Lambda\left(\lambda_{k}\right)+2 \geq$ $2 N\left(\lambda_{k}\right)+3=2 N\left(\lambda_{k+1}\right)+1$, so that (3.4) is true for $r=\lambda_{k+1}$. If $H\left(\lambda_{k+1}\right)=$ 0 , then (3.4) is true for $r=\lambda_{k+1}$ since $\Lambda(r)$ increases by 2 at $r=\lambda_{k+1}$ while $N(r)$ increases by 1 . Finally, suppose $H\left(\lambda_{k}\right)=0, H\left(\lambda_{k+1}\right) \neq 0$. If $H\left(\lambda_{j}\right)=0$ for $j=0,1,2, \cdots, k$, then $\Lambda\left(\lambda_{k+1}\right) \geq \Lambda\left(\lambda_{k}\right) \geq 2 k+3=2 N\left(\lambda_{k+1}\right)+1$, and (3.4) is verified for $r=\lambda_{k+1}$. Otherwise there is a largest $j<k$ for which $H\left(\lambda_{j}\right) \neq 0$, and $\Lambda\left(\lambda_{j}\right) \geq 2 N\left(\lambda_{j}\right)+1$; there are at least $k-j$ zeros of $H(z)$ in $\lambda_{j}<x \leq \lambda_{k+1}$; but the number of zeros in this interval is even if $k-j+1$ is even [ since $H\left(\lambda_{k+1}\right)$ and $H\left(\lambda_{j}\right)$ then have the same sign], odd if $k-j+1$ is odd; so the number of zeros cannot be $k-j$ and hence must be at least $k-j+1$. This completes the proof of (3.4).

By combining (3.4) with (2.1) and (2.2), we see that

$$
\int_{1}^{r} t^{-1} \Lambda(t) d t>2 r-4 \gamma \log r-\text { constant }
$$

where $4 y=2 / p^{\prime}$ if $1 \leq p<\infty, 4 \gamma<2$ if $p=\infty$.

We now appeal to a modification of a result of Levinson [4,pp.7-9] to show that $H(z) \equiv 0$. This is as follows.

LEMmA. Let $\left\{x_{n}\right\}_{-\infty}^{\infty}$ be a sequence of real numbers arranged in nondecreasing order, and let $H(z)$ be an entire function which is known to vanish at all $x_{n}$; if $H(z)$ is known to have a multiple zero at some $x_{n}$, that $x_{n}$ is to be repeated, according to its multiplicity, in the sequence. Let $\nu(r)$ denote the number of $x_{n}$ such that $\left|x_{n}\right| \leq r$ and suppose that

$$
\int_{1}^{r} t^{-1} \nu(t) d t \geq 2 r-\alpha \log r-\text { constant } .
$$

Suppose finally that

$$
|H(x+i y)| \leq\left\{\int_{0}^{\pi / 2} h(t) e^{t|y|} d t\right\}^{2},
$$

where $h(t) \geq 0, h(t) \in L^{p}(0, \pi / 2), 1 \leq p<\infty$. Then $H(z) \equiv 0$ if $\alpha \leq 2 / p^{\prime}$, $p^{\prime}=p /(p-1)$. If $p=\infty$, then $H(z) \equiv 0$ if $\alpha<2$. 
The proof of the lemma is parallel to that given by Boas and Pollard [2] for a similar result, and we omit it.

Since $H(z) \equiv 0$, we have either $F(z) \equiv 0$ or $G(z) \equiv 0$. If $F(z) \equiv 0,(3.3)$ shows that $G\left(\lambda_{2 n+1}\right)=0$; if $G(z) \equiv 0,(3.3)$ shows that $F\left(\lambda_{2 n}\right)=0$.

We first consider the case when $F(z) \equiv 0$. Then, in particular, we have

$$
\int_{-\pi / 2}^{\pi / 2} f(t) d t=0
$$

and

$$
\begin{array}{ll}
\int_{-\pi / 2}^{\pi / 2} f(t) \cos \lambda_{2 n+1} t d t=0 & (n=0,1,2, \cdots), \\
\int_{-\pi / 2}^{\pi / 2} f(t) \sin \lambda_{2 n+1} t d t=0 & (n=0,1,2, \cdots) ;
\end{array}
$$

hence

$$
\int_{-\pi / 2}^{\pi / 2} f(t) e^{i \mu_{n} t} d t=0
$$$$
(n=0, \pm 1, \pm 2, \cdots)
$$

where

$$
\mu_{0}=0, \quad \mu_{n}=\lambda_{2 n-1}(n>0), \quad \mu_{n}=-\lambda_{-2 n-1}(n<0) .
$$

A result of Levinson $[4, p .6]$, reduced to the interval $(-\pi / 2, \pi / 2)$, is that $\left\{e^{i \mu_{n} t}\right\}$ is $L^{p}$-complete if $M(t)$, the number of $\left|\mu_{n}\right| \leq t$, satisfies

$$
\int_{1}^{r} t^{-1} M(t) d t>r-\left(1 / p^{\prime}\right) \log r-\text { constant }
$$

$1 \leq p<\infty$; his proof also shows that $L^{\infty}$-completeness follows from (3.8) if $1 / p^{\prime}$ is replaced by any number less than 1 . Since $M(t)=2 N_{2}(t)+1$, (3.8) is true in virtue of (2.2). Thus (2.2) implies $f(t)=0$ almost everywhere if $F(z) \equiv 0$.

Now suppose that $G(z) \equiv 0$. In the same way we have

$$
\int_{-\pi / 2}^{\pi / 2} f(t) e^{i \mu_{n} t} d t=0
$$

where now

$$
\mu_{n}=\lambda_{2 n}(n \geq 0), \quad \mu_{n}=-\lambda_{-2 n-2}(n<0)
$$


In this case $M(t)=2 N_{1}(t)$ and (3.8) follows from (2.1). The rest of the argument is as before.

4. Proof of Corollary 1. To prove Corollary 1 we have to show that (2.1) and (2.2) follow from $0 \leq \lambda_{n} \leq n+\delta(n=0,1,2, \cdots)$, where $\delta=1+1 / p^{\prime}$, $1 \leq p<\infty$. In the interval $2 k+\delta \leq u<2 k+\delta+2$, where $k=0,1,2, \cdots$, we have $N_{1}(u) \geq k+1$. Let $x>1$ and define $n$ by $2 n+\delta \leq x<2 n+\delta+2$. Then

$$
\begin{aligned}
\int_{\delta}^{x} \frac{N_{1}(u)}{u} d u & \geq \int_{\delta}^{2+\delta} \frac{d u}{u}+\int_{2+\delta}^{4+\delta} \frac{2 d u}{u}+\cdots+\int_{2 n-2+\delta}^{2 n+\delta} \frac{n}{u} d u \\
& =\sum_{k=1}^{n} k \log \left(1+\frac{2}{2 k+\delta-2}\right) \\
& \geq \sum_{k=1}^{n} k\left\{\frac{2}{2 k+\delta-2}-\frac{1}{2}\left(\frac{2}{2 k+\delta-2}\right)\right\} \\
& \geq \sum_{k=2}^{n}\left\{1+\frac{2-\delta}{2 k}-\frac{1}{2(k-1)}\right\} \\
& =n+\left(1-\frac{1}{2} \delta-\frac{1}{2}\right) \log n+O(1) \\
& =\frac{1}{2} x+\frac{1-\delta}{2} \log x+O(1)=\frac{1}{2} x-\frac{1}{2 p^{\prime}} \log x+O(1) .
\end{aligned}
$$

On the other hand, in the interval $2 k+1+\delta \leq u<2 k+3+\delta(k=0,1,2, \cdots)$, we have $N_{2}(u) \geq k+1$. Thus

$$
\begin{aligned}
\int_{1}^{x} \frac{N_{2}(u)}{u} d u & \geq \int_{1+\delta}^{3+\delta} \frac{d u}{u}+\cdots+\int_{2 n-1+\delta}^{2 n+1+\delta} \frac{n}{u} d u \\
& =\sum_{k=1}^{n} k \log \left(1+\frac{2}{2 k-1+\delta}\right) \\
& \geq \sum_{k=1}^{n} k\left\{\frac{2}{2 k-1+\delta}-\frac{1}{2}\left(\frac{2}{2 k-1+\delta}\right)^{2}\right\}
\end{aligned}
$$




$$
\begin{aligned}
& \geq \sum_{k=1}^{n}\left\{1+\frac{1-\delta}{2 k+1}-\frac{1}{2 k-1}\right\} \\
& =x+\frac{1}{2}(1-\delta)-\frac{1}{2}+O(1) \\
& =x-\frac{1}{2 p^{\prime}}-\frac{1}{2}+O(1) .
\end{aligned}
$$

5. Proof of Corollaries 2-5. In proving Corollaries 2-5, it is convenient to write $-a_{n}$ instead of $a_{n}$, and $t=x-\pi / 2$, so that (2.3) becomes

$\begin{cases}\cos \left(\lambda_{n} x-\lambda_{n} \pi / 2\right)-a_{n} \sin \left(\lambda_{n} x-\lambda_{n} \pi / 2\right) & (n \text { even }) ; \\ a_{n}\left(\cos \lambda_{n} x-\lambda_{n} \pi / 2\right)+\sin \left(\lambda_{n} x-\lambda_{n} \pi / 2\right) & (n \text { odd }) .\end{cases}$

Put $a_{n}\left(1+a_{n}^{2}\right)^{-1 / 2}=\sin b_{n},\left(1+a_{n}^{2}\right)^{-1 / 2}=\cos b_{n}, 0 \leq b_{n}<\pi / 2$ or $-\pi / 2<$ $b_{n} \leq 0$, according as $a_{n} \geq 0$ or $a_{n} \leq 0$. Then the completeness of (2.3) is equivalent to that of

$$
\begin{cases}\cos \left(\lambda_{n} x-\lambda_{n} \pi / 2\right) \cos b_{n}-\sin \left(\lambda_{n} x-\lambda_{n} \pi / 2\right) \sin b_{n} & (n \text { even }) ; \\ \sin \left(\lambda_{n} x-\lambda_{n} \pi / 2\right) \cos b_{n}+\cos \left(\lambda_{n} x-\lambda_{n} \pi / 2\right) \sin b_{n} & (n \text { odd }) ;\end{cases}
$$

that is, to the completeness of

$$
\left\{\begin{array}{lr}
\cos \left(\lambda_{n} x-\lambda_{n} \pi / 2+b_{n}\right) & (n \text { even }) ; \\
\sin \left(\lambda_{n} x-\lambda_{n} \pi / 2+b_{n}\right) & (n \text { odd }) .
\end{array}\right.
$$

Now let $\lambda_{n}=m-2 \epsilon_{n} / \pi$, where $m$ is an integer of the same parity as $n$. Then the completeness of (2.3) is equivalent to that of

$$
\cos \left(\lambda_{n} x+\epsilon_{n}+b_{n}\right) \quad(n=0,1,2, \cdots) .
$$

Thus a set

$$
\cos \left(\lambda_{n} x+q_{n}\right)
$$

is equivalent to a set of the form (2.3) if for all $n$ either

$$
\epsilon_{n} \leq q_{n}<\pi / 2+\epsilon_{n}
$$


or

$$
-\pi / 2+\epsilon_{n}<q_{n} \leq \epsilon_{n} \text {. }
$$

We may satisfy (5.3) or (5.4) in various ways. For example, (5.3) is certainly true if $\left|n-\lambda_{n}\right|<\delta(n=0,1,2, \cdots)$, with $\delta<1 / 2$ and $\pi \delta / 2 \leq q_{n}<$ $\pi(1-\delta) / 2$; this establishes Corollary 2, since the condition of Corollary 1 is certainly satisfied in this case. Corollary 1 requires only that $\lambda_{n} \leq n+1$ if $p=1$; if we restrict $\lambda_{n}$ to lie always on one side of $n$ we can therefore obtain a stronger result than Corollary 2. In fact, if $n \leq \lambda_{n} \leq n+1$ we have $\epsilon_{n} \leq 0$, and (5.3) is satisfied if $0 \leq q_{n}<\tau / 2+\epsilon_{n}$, hence certainly if $n \leq \lambda_{n} \leq$ $n+\delta, \delta<1$, and $0 \leq q_{n}<\pi(1-\delta) / 2$. On the other hand, if $n-1 \leq \lambda_{n} \leq n$ $(n>0)$, we have $\epsilon_{n} \geq 0$ and (5.4) is satisfied if $n-\delta \leq \lambda_{n}<n(n>0)$, $\delta<1$, and $-\pi(1-\delta) / 2<q_{n} \leq 0$.

If we let $\lambda_{n}=m-2 \epsilon_{n} / \pi$, where $m$ has opposite parity to $n,(2.3)$ reduces to $\left\{\sin \left(\lambda_{n} x+\epsilon_{n}+b_{n}\right)\right\}$; by taking $m=n+1$ we obtain Corollary 4. Finally, Corollary 5 is obtained by taking $m=n+2$. Further theorems of the same character are readily written down.

\section{REFERENCES}

1. A. V. Bitsadze, $O b$ odnoì sisteme funktsiĩ [On a system of functions], Uspehi Matem. Nauk (N.S.) 5, no. 4 (38) (1950), 154-155.

2. R. P. Boas, Jr., and H. Pollard, Complete sets of Bessel and Legendre functions, Ann. of Math. (2) 48 (1947), 366-384.

3. V. A. Ditkin, O polnote odnoì sistemi trigonometricheskih funktsii [On the completeness of a system of trigonometric functions.], Uspehi Matem. Nauk (N.S.) 5, no. 2 (36) (1950), 196-197.

4. N. Levinson, Gap and density theorems, Amer. Math. Soc. Colloquium Publications, vol. 26; American Mathematical Society, New York, 1940.

5. R.E. A.C. Paley and N. Wiener, Fourier transforms in the complex domain, Amer. Math. Soc. Colloquium Publications, vol. 19; American Mathematical Society, New York, 1934.

Northwestern University 


\title{
PACIFIC JOURNAL OF MATHEMATICS
}

\section{EDITORS}

\author{
HERBERT BUSEMANN \\ University of Southern California \\ R. M. RoBINSON \\ Los Angeles 7, California \\ University of California \\ Berkeley 4, California \\ E. F. BECKENBACH, Managing Editor \\ University of California \\ Los Angeles 24, California
}

\section{ASSOCIATE EDITORS}

R. P. DILWORTH
HERBERT FEDERER
MARSHALL HALL

\author{
P. R. HALMOS \\ HEINZ HOPF \\ R. D. JAMES
}

\author{
B $\emptyset$ RGE JESSEN \\ PAUL LÉVY \\ GEORGE PÓLYA
}

\author{
J. J. STOKER \\ E. G. STRAUS \\ KÔSAKU YOSIDA
}

\section{SPONSORS}

UNIVERSITY OF BRITISH COLUMBIA

CALIFORNIA INSTITUTE OF TECHNOLOGY

UNIVERSITY OF CALIFORNIA, BERKELEY

UNIVERSITY OF CALIFORNIA, DAVIS

UNIVERSITY OF CALIFORNIA, LOS ANGELES

UNIVERSITY OF CALIFORNIA, SANTA BARBARA

OREGON STATE COLLEGE

UNIVERSITY OF OREGON

\author{
UNIVERSITY OF SOUTHERN CALIFORNIA \\ STANFORD UNIVERSITY \\ WASHINGTON STATE COLLEGE \\ UNIVERSITY OF WASHINGTON

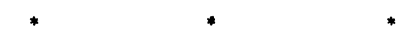 \\ AMERICAN MATHEMATICAL SOCIETY \\ NATIONAL BUREAU OF STANDARDS, \\ INSTITUTE FOR NUMERICAL ANALYSIS
}

Mathematical papers intended for publication in the Pacific Journal of Mathematics should be typewritten (double spaced), and the author should keep a complete copy. Manuscripts may be sent to any of the editors. All other communications to the editors should be addressed to the managing editor, E. F. Beckenbach, at the address given above.

Authors are entitled to receive 100 free reprints of their published papers and may obtain additional copies at cost.

The Pacific Journal of Mathematics is published quarterly, in March, June, September, and December. The price per volume (4 numbers) is $\$ 8.00$; single issues, $\$ 2.50$. Spécial price to individual faculty members of supporting institutions and to members of the American Mathematical Society: $\$ 4.00$ per volume; single issues, $\$ 1.25$.

Subscriptions, orders for back numbers, and changes of address should be sent to the publishers, University of California Press, Berkeley 4, California.

UNIVERSTTY OF CALIFORNIA PRESS • BERKELEY AND LOS ANGELES 


\section{Pacific Journal of Mathematics}

\section{Vol. 1, No. $3 \quad$ BadMonth, 1951}

R. P. Boas, Completeness of sets of translated cosines ............. 321

J. L. Brenner, Matrices of quaternions . ..................... 329

Edmond Darrell Cashwell, The asymptotic solutions of an ordinary differential equation in which the coefficient of the parameter is

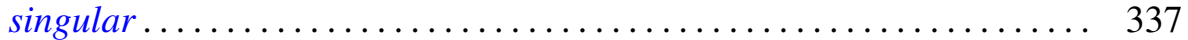

James Dugundji, An extension of Tietze's theorem ................ 353

John G. Herriot, The polarization of a lens ...................... 369

J. D. Hill, The Borel property of summability methods ............... 399

G. G. Lorentz, On the theory of spaces $\Lambda \ldots \ldots \ldots \ldots \ldots \ldots \ldots \ldots \ldots . \ldots \ldots$

J. H. Roberts and W. R. Mann, On a certain nonlinear integral equation of the Volterra type ................................. 431

W. R. Utz, A note on unrestricted regular transformations . . .......... 447

Stanley Simon Walters, Remarks on the space $H^{p} \ldots \ldots \ldots \ldots \ldots \ldots . . \ldots 55$

Hsien Chung Wang, Two theorems on metric spaces ............... 473 